\title{
Hardware Design of Combustion Control System for Small-size Circulation Fluidized Bed Boiler
}

\author{
Jun Liu ${ }^{\mathrm{a}}$, Jing Chen ${ }^{\mathrm{b}}$, Youxin Yuan ${ }^{\mathrm{c}}$, Zhuo Chen ${ }^{\mathrm{d}}$ \\ School of Automation, Wuhan University of Technology, Wuhan China 430070 \\ aemail:373660860@qq.com, bemail:jingchen680@163.com, cemail:yyx2000@263.net,
}

\begin{abstract}
Keywords: Small-size circulation fluidized bed boiler; Combustion control; Distributed control; Hardware design
\end{abstract}

\begin{abstract}
Traditional old boiler is energy-intensive and highly polluted. Circulation fluidized bed boiler (CFBB) is characterized by environment-friendly, high efficiency and energy-saving. Hardware of Combustion control system for small-size circulation fluidized bed boiler is presented in this paper to transform the old boiler. It is a three-tier structure and distributed control system for small-size circulation fluidized bed boiler. The following works have been down in the study: introduce the work of the small-size CFBB, propose the control requirements of small-size CFBB combustion control system, design the hardware of the small-size CFBB combustion control system. The hardware circuit designed in this paper has been successfully applied to the small-size CFBB system. It can improve the automatic control level and combustion efficiency, complete energysaving and emission reduction.
\end{abstract}

\section{Introduction}

With the rapidly development of national industry, the environment pollution and haze problem are becoming more and more serious. Circulating fluidized bed boiler starts earlier and upgrades technology faster abroad. And it is rapidly assembled. The market share of old inefficient boiler is big in domestic, and to reduce the investment, the companies use the upgraded boiler[1].

Based on the small-size CFBB combustion process, the control requirements of the boiler has been analyzed. The input and output variables of small CFBB are detected in order to control the boiler better[2]. Accounting for the control requirements, the distributed control system is been selected[3]. Combining with field test platform, the carding, analysis and the researching on combustion system control tasks, the hardware design of small-size circulation fluidized bed boiler distributed combustion control system is completed. Therefore, the hardware of combustion control system for small-size circulation fluidized bed boiler is presented in this paper.

In the presented boiler, the fuels are combusted boiling under the action of air in the furnace bottom. It is mixed a certain percentage of desulfurizer. he solid particles that are not burned return to combust through efficient cyclone separation.

\section{Control Requirements}

Based on small-size CFBB combustion technology, the input and output variables from searching control requirements of the system are proposed. The input and output variables of the combustion control system diagram is shown in Fig.1.

Input variables of the boiler: Feed-water quantity, attemperate water quantity, feed-coal quantity, supply air rate, induced air rate and returns rate.

Output variables of the boiler: Drum water level, steam temperature, steam pressure, furnace temperature, oxygen content of fume (excess air coefficient $\alpha$ ) and furnace negative pressure. 


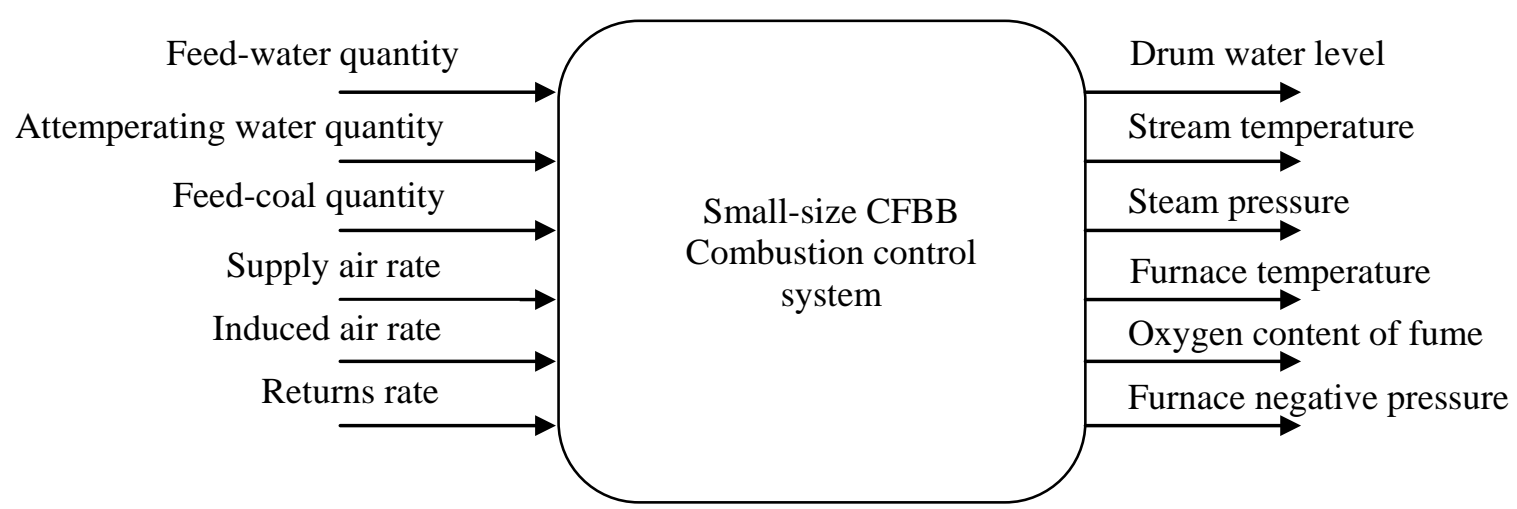

Fig.1. Input and output variables of the combustion control system diagram

According to the coupling relationship between variables, the control system is separated into two system: combustion system and steam-water system. For better control the CFBB combustion, combustion system and steam-water system are divided into several subsystems[4]. The control objects of the CFBB are shown in Fig.2.

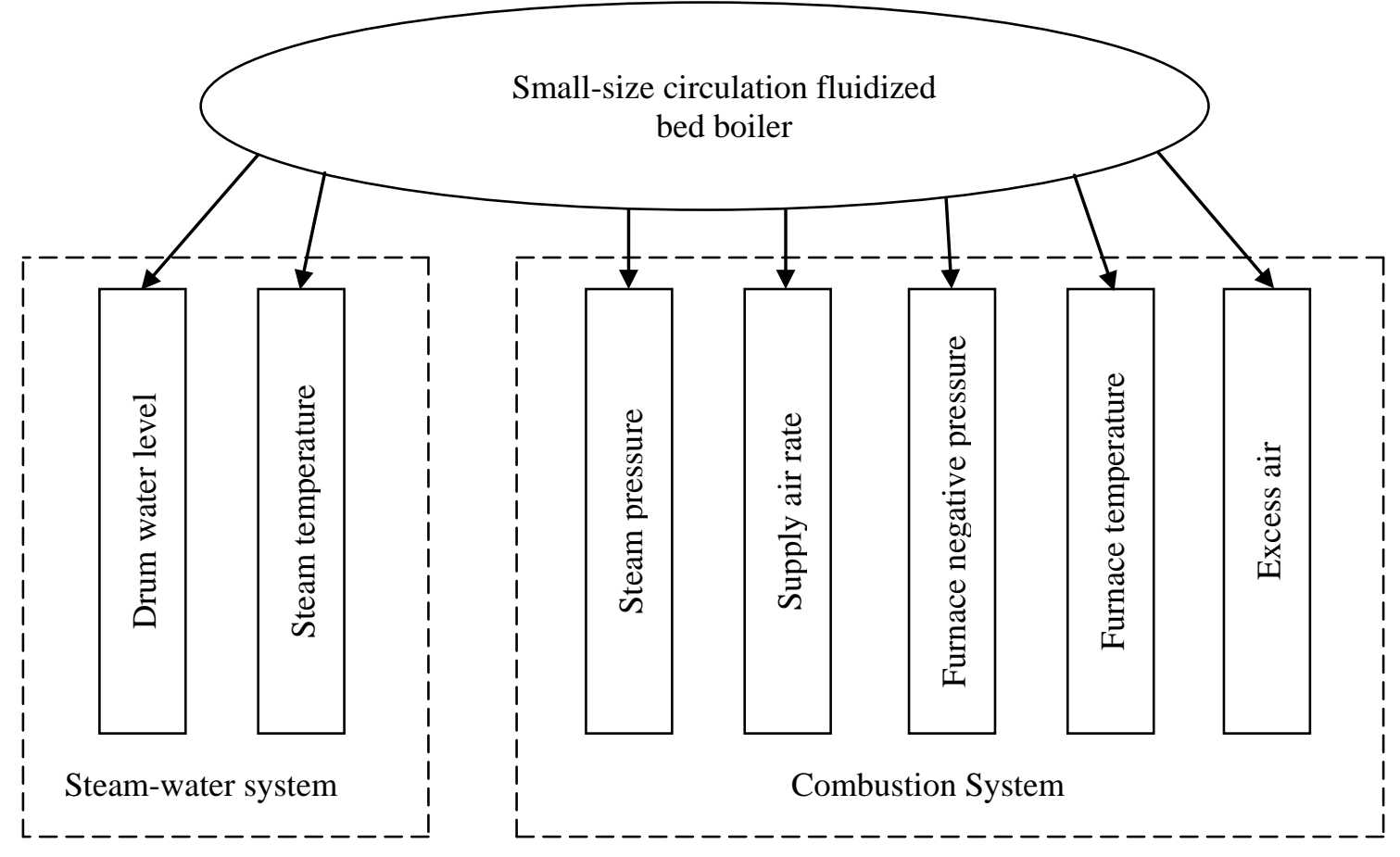

Fig.2. Control objects of the CFBB

\section{Hardware Design}

The hardware design of the small-size CFBB combustion control system mainly includes the detection circuit of process variables, single conditioning and sampling, control devices, actuators and host computer[5]. The structure of the CFBB combustion control system is shown in Fig.3. The system is a three-tier structure and distributed control system.

The detection circuit of process variables. The circuits can detect furnace temperature, stream pressure, furnace negative pressure, oxygen content of fume and so on. Through the measurement principle of the process variables and work environment, the appropriate sensors are chosen for indepth study. The sensors convert non-electricity signals to electrical.

Single conditioning and collecting circuits. The circuits include amplifying and adjustment circuit of thermocouple signals, I/V converting circuit and A/D converter circuit.

Controllers. DSP and Microcontroller are the workshop controllers. PLC is the upper controller in the distributed control system. The workshop controllers consist of the minimum system and 
peripheral circuit, such as crystal oscillator, power source, reset circuit, ADC and communication interface circuit.

Actuator. The actuator includes blower, induced draft fan, coal feeder with scale system and inverter. Control command is transmitted to the actuator by Modbus protocol. Then furnace temperature, negative pressure and stream pressure can keep stable by adjusting blast volume and coal feed rate properly.

Host computer. The host computer is connected to the PLC by RS485 interface. Monitoring system and setting parameters can be implemented by the touch screen.

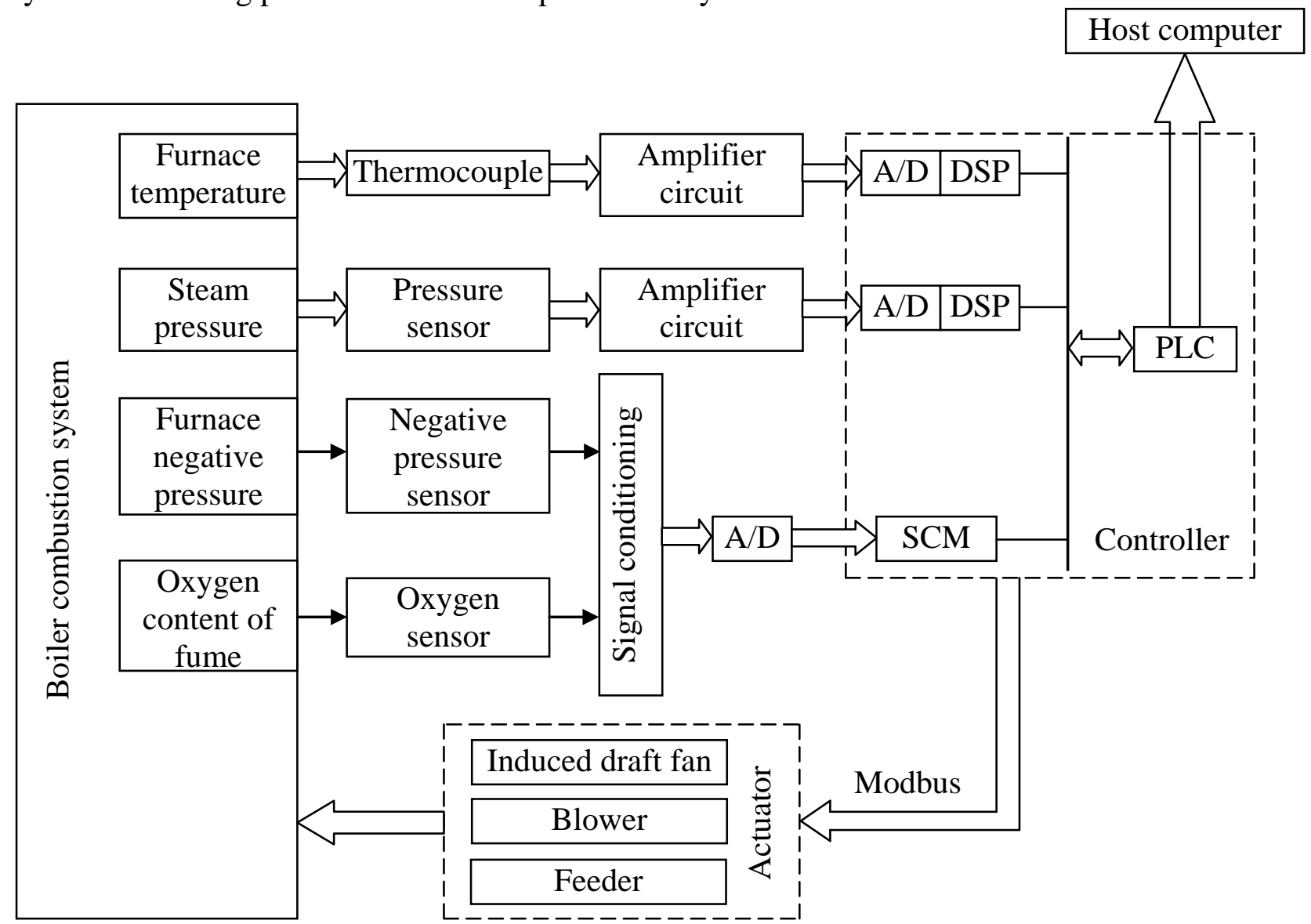

Fig.3. Structure of the CCFB combustion control system

\section{Conclusions}

Circulation fluidized bed boiler is characterized by environment-friendly, high efficiency and energy-saving. Hence, hardware of Combustion control system for small-size circulation fluidized bed boiler is presented in this paper to transform the old boiler. The following works have been done in the study: introduce the work of the small-size CFBB, propose the control requirements of small-size CFBB combustion control system, design the hardware of the small-size CFBB combustion control system. The hardware circuit designed in this paper has been successfully applied to the small-size CFBB system. It can improve the automatic control level and combustion efficiency of the combustion control system for small-size CFBB, complete energy-saving and emission reduction.

\section{Acknowledgement}

The paper is supported by the projects of Scientific Support Plans of Hubei Province (Granted No: 2013BAA009). 


\section{References}

[1] Xiaohui Wang, Qin Dong, Zhengbin Tian, Present Situation and Development Prospect of the Circulating Fluidized Bed Boiler Technology [J]. Energy Research and Information, 200622 (3), 165-168.

[2] Yongjin Yin, Yuliang Qi, Some Thoughts of Transforming Chain-grate Boiler into CFBB [J]. Industrial Boiler, 200730 (4), 33-34.

[3] Ming Li, Xiangdong Xu, Circulating Fluidized Bed Boiler Control System [J]. Journal of Tsinghua University (Science and Technology), 20025 (42), 665-668.

[4] Leming Cheng, Qiang Zhou, Zhenglun Shi, Design and Operation of Circulating Fluidized Bed Boiler Transformed from Chain-gate Boiler [J]. Thermal Power Generation, 200534 (3), 25-27.

[5] Rychkov Alexander D., Filipov Konstantin P., Numerical Modeling of Coal Combustion Processes in Ecological Clear Circulating Fluidized Bed Boiler Units[C]. Science and Technology, 2000, 304-309. 